\title{
Reaction Force Compensator for High-Speed Precision Stage of Laser Direct Imaging Process
}

\author{
Chang-hoon Seo $\left(\mathbb{D},{ }^{1}\right.$ Yong ho Jeon $\left(\mathbb{D},{ }^{1}\right.$ Hyung-ku Lee $\left(\mathbb{D},{ }^{2}\right.$ \\ Hyo-young Kim $\mathbb{D}^{\mathrm{D}}{ }^{3}$ and Moon G. Lee $\mathbb{D}^{1}$ \\ ${ }^{1}$ Department of Mechanical Engineering, Ajou University, Suwon 16499, Republic of Korea \\ ${ }^{2}$ LEETECH Co., Ltd., Hwaseong 18255, Republic of Korea \\ ${ }^{3}$ Manufacturing System ReD Group, Korea Institute of Industrial Technology, Cheonan 31056, Republic of Korea \\ Correspondence should be addressed to Moon G. Lee; moongulee@ajou.ac.kr
}

Received 27 November 2017; Revised 23 April 2018; Accepted 27 May 2018; Published 19 June 2018

Academic Editor: Francesco Franco

Copyright (C) 2018 Chang-hoon Seo et al. This is an open access article distributed under the Creative Commons Attribution License, which permits unrestricted use, distribution, and reproduction in any medium, provided the original work is properly cited.

Recently, laser direct imaging (LDI) process has become popular as a substitute for lithography in flexible printed circuit board (FPCB) manufacturing industry. However, repeated motion of the process equipment causes residual vibration in a transient state when the stage accelerates or decelerates. The supporting structure for the laser head is complex and heavy in order to increase the resonant frequency, because the residual vibration must be controlled below a certain level for the LDI process precision. If the vibration cannot be rejected, the controller needs longer settling time; therefore, the productivity is reduced. In this study, a reaction force compensator (RFC) for the granite of a precision stage in the equipment is proposed to minimize the detrimental vibration and steady state error. First, displacement is measured with a laser Doppler vibrometer (LDV) to identify the dynamic characteristics of a pneumatic isolator for the stage. Second, the compensator's mechanism design is proposed by using a voice coil motor and capacitive displacement sensor. Third, a hybrid control algorithm combining the RFC and PID is applied to reduce the vibration. Finally, the RFC is evaluated in terms of vibration peak and steady state error. The LDI apparatus is stabilized more rapidly, because the proposed method cancels the impulsive reaction force of linear motion module immediately.

\section{Introduction}

Laser direct imaging (LDI) is highlighted as an alternative process to lithography. Lithography using masks has been widely applied for circuit patterning in conventional flexible printed circuit board (FPCB) manufacturing processes. However, it is difficult to satisfy the requirements of batch production. This is because manufacturing processes using masks involve complicated procedures and additional costs incurred by mask replacements [1]. Furthermore, an ultraprecise stage with submicron accuracy is required for alignment between the mask and workpiece [2-4]. Thus, LDI processing has recently emerged as an alternative method [5-7]. When using the exposure equipment with a UV laser as a light source, a circuit pattern with a minimum line width of $5-10 \mu \mathrm{m}$ is fabricated. In the process, a step-and-scan motion with large acceleration and deceleration of the optical head or workpiece is mainly applied.
The parasitic vibration and displacement error in the workpiece caused by the step-and-scan motion must be reduced. The linear motion module in the stage on a granite base transports the workpiece. The motion has reciprocating long travel to manufacture printed circuit pattern on a large flexible substrate. The workpiece is transported at high speed to increase productivity. During the acceleration and deceleration intervals, unwanted displacement of the granite occurs on its vibration isolator due to the low stiffness of its air spring and the reaction force from the linear motion module.

The motion of the granite caused the parasitic vibration of the support structure for the optical components of the UV laser head and workbench. To prevent resonance that is originated from the motion of the granite, the support structure must be made larger and heavier. A tendency toward miniaturization and lighter weight is one of the major trends in the industry; however, the heavy UV laser head is a constraint to design a smaller manufacturing system. This is 


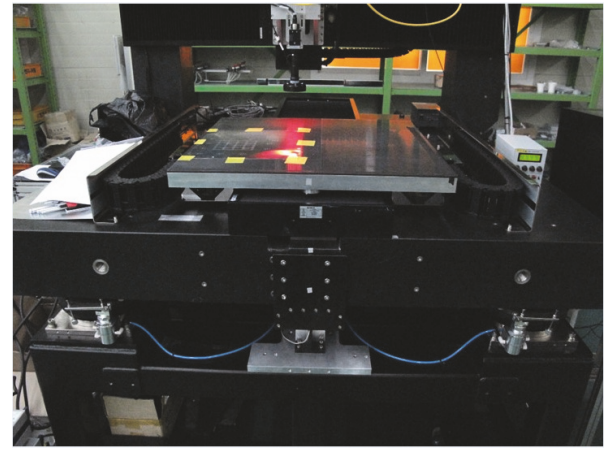

FIGURE 1: Precision stage for laser direct imaging (LDI) process.

why a reaction force compensator is needed for LDI process even though there is no specified requirement in terms of vibration level.

The periods of acceleration and deceleration have short duration but large magnitude. Therefore, the reaction force applied to the granite has characteristics similar to that of the impulsive force. The force causes parasitic vibration and displacement error, which eventually result in unwanted error on the stage and workpiece [8-10]. Therefore, it is necessary to compensate for the reaction force to prevent the vibration and error on the granite and to increase the stability of the system with respect to operations at the high acceleration and deceleration speeds. Figure 1 shows the LDI stage that is operated in step-and-scan mode.

An input shaping method to prevent the system from vibrating is commonly used because the resonating frequency component in motion profile can be easily derived and rejected if a user designates the profile [11]. However, it is not possible to consistently ensure successive vibration reduction performance due to the complex structure, various motion profiles, and random servo-actuation. A feedforward control to cancel the harmonic disturbance using an adaptive gain scheme was suggested by Pin et al. [12]. Matsuda et al. proposed a reaction disturbance cancellation algorithm based on disturbance observer using a displacement sensor [13]. Kim et al. proposed a modified feedforward algorithm to control lateral vibration of the granite on the pneumatic mounts with a magnetorheological damper system [14]. However, they have needed additional sensors and shown limited performance like slow response.

In this study, a reaction force compensator (RFC) with voice coil motors (VCMs) is proposed and designed to offset the disadvantages caused by the low stiffness of the pneumatic vibration isolator. The design is based on the dynamic characteristics of a precision stage with a pneumatic vibration isolator. The motivation of this research is to improve the existing equipment with minimum modification of mechanism and controller. Therefore, a control algorithm that suppresses the parasitic vibration and displacement error of the granite by using a simple gain related to the thrust force of the linear motor is also proposed. The proposed control algorithm can be used without any vibration sensor. A feedback control may optionally be used to increase
TABLE 1: Specifications of the precision stage for the LDI process.

\begin{tabular}{lccc}
\hline Item & Unit & X-axis & Y-axis \\
\hline Rated stroke & $\mathrm{mm}$ & 600 & 600 \\
Maximum velocity & $\mathrm{mm} / \mathrm{s}$ & 1,000 & 1,000 \\
Maximum acceleration & $\mathrm{G}$ & 1.0 & 1.0 \\
Repeatability & $\mu \mathrm{m}$ & 2.0 & 2.0 \\
Accuracy & $\mu \mathrm{m}$ & 3.0 & 3.0 \\
\hline
\end{tabular}

TABle 2: Specifications of the linear motion module for X-axis.

\begin{tabular}{lcc}
\hline Item & Unit & Y-axis \\
\hline Rated speed & $\mathrm{mm} / \mathrm{s}$ & 1,500 \\
Peak speed & $\mathrm{mm} / \mathrm{s}$ & 1,500 \\
Rated force & $\mathrm{N}$ & 325 \\
Instantaneous peak force & $\mathrm{N}$ & 1,300 \\
\hline
\end{tabular}

the performance of the reaction force compensation when needed. The algorithm was verified and evaluated through a series of experiments.

\section{Dynamic Characteristics of Precision Stage with Passive Pneumatic Vibration Isolator}

In order to design the RFC, the operation condition of the precision stage for LDI must be understood, and the dynamic characteristics of the stage must be identified. The repetitive operation of the stage's linear motion module incurs the parasitic motion of the stage. Figure 2(a) shows the force and velocity of the linear motion module in the time domain using the reference trajectory of the precision stage that is presented in Figure 1. A general specification of the precision stage is given in Table 1 . It is assumed that the linear motion module is accelerated and decelerated with $0.5 \mathrm{G}$ along the $\mathrm{X}$-axis. In this $0.5 \mathrm{G}$ interval, the motion profile exhibits the maximum acceleration and maximum speed, which are the worst conditions like impulsive force.

Table 2 shows the specifications of the linear motion module used in the precision stage as illustrated in Figure 1. The output of the linear motion module in the acceleration and deceleration interval at $0.5 \mathrm{G}$ corresponded to $83 \%$ of the rated force, and this corresponded to a thrust of 270 N. An instantaneous force generated by servo control in the interval could not exceed the $325 \mathrm{~N}$ of thrust force that corresponded to the rated maximum force of the module. The output force and velocity components that were collected from the linear motion are shown in Figure 2(a).

In order to identify the dynamic characteristics, especially, the response of the granite on the passive vibration isolator, the motion of the granite was measured under excitation by the reaction force of the linear motion module along a reference trajectory by using a displacement sensor. The long stroke motion in Figure 2(a) was repeatedly engaged in measuring the displacement that occurred on the granite by using a laser Doppler vibrometer (LDV, Polytec OFV5000). The LDV gathers the displacement and vibration the frequency range of interest, which was then analyzed by 


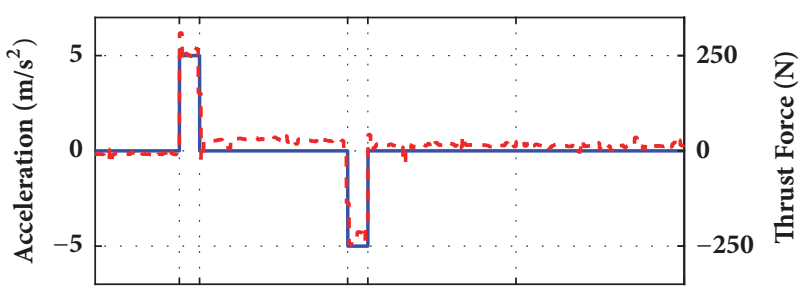

- Reference Signal

- - - Measured Force

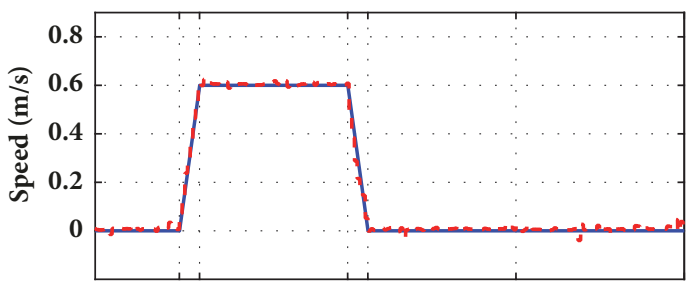

— Reference Signal

- - - Measured Speed

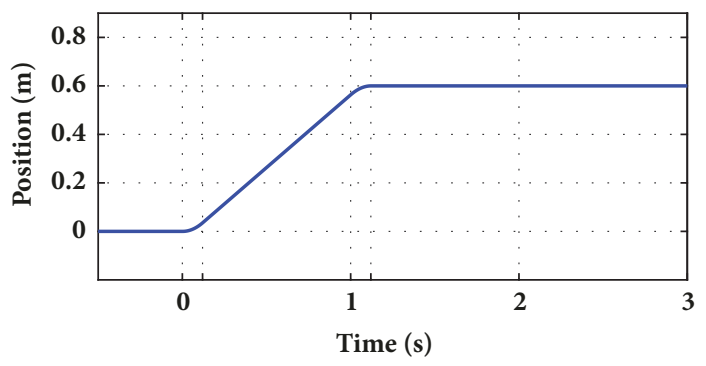

— Reference Signal

(a)

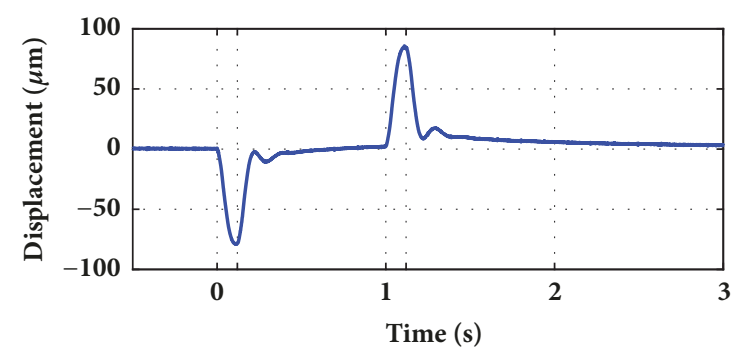

Granite

(b)

FIGURE 2: Movement of (a) linear motion module and (b) granite with a single step-and-scan mode.

spectrum analysis using a dynamic signal analyzer (DSA, Agilent 35670A).

Figure 2(b) shows the measured displacement signal. Some features are observed. First, there are two sharp peaks of approximately $86 \mu \mathrm{m}$ synchronized with the acceleration and deceleration interval. The peak displacements occur instantaneously due to the reaction force of the linear motion module at the interval. Second, smooth $10 \mu \mathrm{m}$ vibrations were also seen. This is because the vibration isolator attempts to

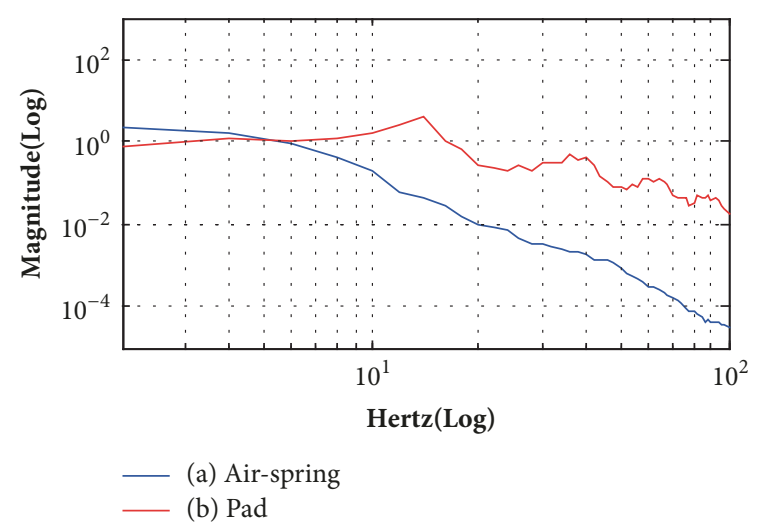

FIGURE 3: Conventional vibration isolator's frequency response with (a) air spring and (b) NBR antivibration pad.

maintain the granite at its original orientation and position by the pneumatic valve working in the isolator. The isolator rotates and moves the granite a little, when the granite is tilted from the horizontal plane and deviated from its initial location by the change of system's center of mass after the motion. This occurred continuously while the linear motion module moved, and the peaks tended to increase if the motion involved large acceleration of a long stroke at high speed.

A conventional passive pneumatic vibration isolator has insufficient attenuation in the low frequency range. Typically, passive air springs or antivibration pads with viscoelastic materials are used in precision systems to reduce microvibrations because they have low stiffness and high damping ratios. The low stiffness creates a low natural frequency, which provides effective vibration reduction in the high frequency range. The high damping ratio results in good attenuation adjacent to the natural frequency. Despite these characteristics, the passive vibration isolator still does not perform well at very low frequencies below $5 \mathrm{~Hz}$, because it does not have a sufficiently small natural frequency.

An LDV and a DSA were used to characterize and investigate the dynamic response of the granite, which consisted of an air spring isolator or an antivibration pad in the same frequency range. The pad was made of nitrile butadiene rubber (NBR), which is widely used in industry. In the graph shown in Figure 3, the horizontal resonant frequencies of the precision stage with air spring isolator and that with the NBR antivibration pad are approximately $2.5 \mathrm{~Hz}$ and 10.5 $\mathrm{Hz}$, respectively. Even when using the air spring, the isolator could not reduce the vibration in the frequency range to less than its own natural frequencies. The reaction force as a source of vibration possessed characteristics identical to the thrust force of the linear motor in Figure 2(a), albeit in the opposite direction.

The high amplitude vibration in the low frequency range needs to be diminished. The above measurements indicated that the reduction effect of the air spring in frequency ranges exceeding $11.0 \mathrm{~Hz}$ was approximately 3 times more effective than that of the NBR pad isolator. However, the insufficient attenuation due to the low stiffness of the air spring isolator in frequency ranges below $5.0 \mathrm{~Hz}$, which includes rigid 
body motion, still needs improvement. The reaction force of the linear motion applied to the granite included frequency components below $5.0 \mathrm{~Hz}$. Hence, it was necessary to improve a vibration isolator with low transmissibility in a wider region by properly compensating for the aforementioned components in the range below $5.0 \mathrm{~Hz}$.

\section{Mechanical Design for Reaction Force Compensator}

A novel RFC is designed to actively attenuate the disturbance in the low frequency range from the linear motion. The RFC is composed of a metallic bracket and two separate modules. Each separate module includes unidirectional VCMs and noncontact displacement sensors. The bracket is mounted underneath the granite. The driving force that is generated in the VCM is transferred to the granite through the bracket. There are two compensator modules, namely, the forward(F-) module and backward- (B-) module. The modules were arranged in opposite directions with a preload. This arrangement created forward and backward thrust.

When setting up the VCMs, there were some considerations because the gap between the coil and the magnet in the VCMs is small. The gap is vertical to the VCM's drive direction ( $\mathrm{x}$-axis). Therefore, the parasitic force along the $y$ - and z-axes should not be transferred. A point-contact mechanism between the bracket and the VCM is introduced by using a ball caster to prevent damage due to unexpected parasitic forces. The magnet in the VCM was fixed to the lower frame with high stiffness, and the ball caster was mounted in the coil assembly along with the linear motion (LM) guide. The bracket and ball keep the contact without separation despite the VCM motion because the preload is engaged by applying an offset DC current to the VCMs.

The RFC with two modules has a symmetric planar configuration along the axis that is parallel to the linear motion. Each VCM has unidirectional operation in contrast to the single actuator scheme with only one VCM that covers the full stroke. Generally, if only a single actuator is adopted, it becomes nonsymmetric and heavy. For the large force requirement, the module can be expensive. The symmetricity provides robustness with respect to the thermal error and enables easy control of the system. The proposed configuration includes advantages when developing a modular design for a multiaxes combination with limited space and a complex structure.

The granite displacement was continuously monitored through noncontact displacement sensors that was installed in the F- and B-modules, separately. This was used to perform feedback control and to suppress effectively unpredictable movement by the isolator valve working. The arrangement of the main components and detailed sectional views of the modules are shown in Figure 4. A steel bracket guide mechanism is omitted to enable visibility of assembled components in Figure 4(b).

The required actuating force of the VCM was estimated by summing the reaction forces based on the thrust force of the linear motor and the inertial force from the movement of the granite. After the estimation, the VCMs are selected to

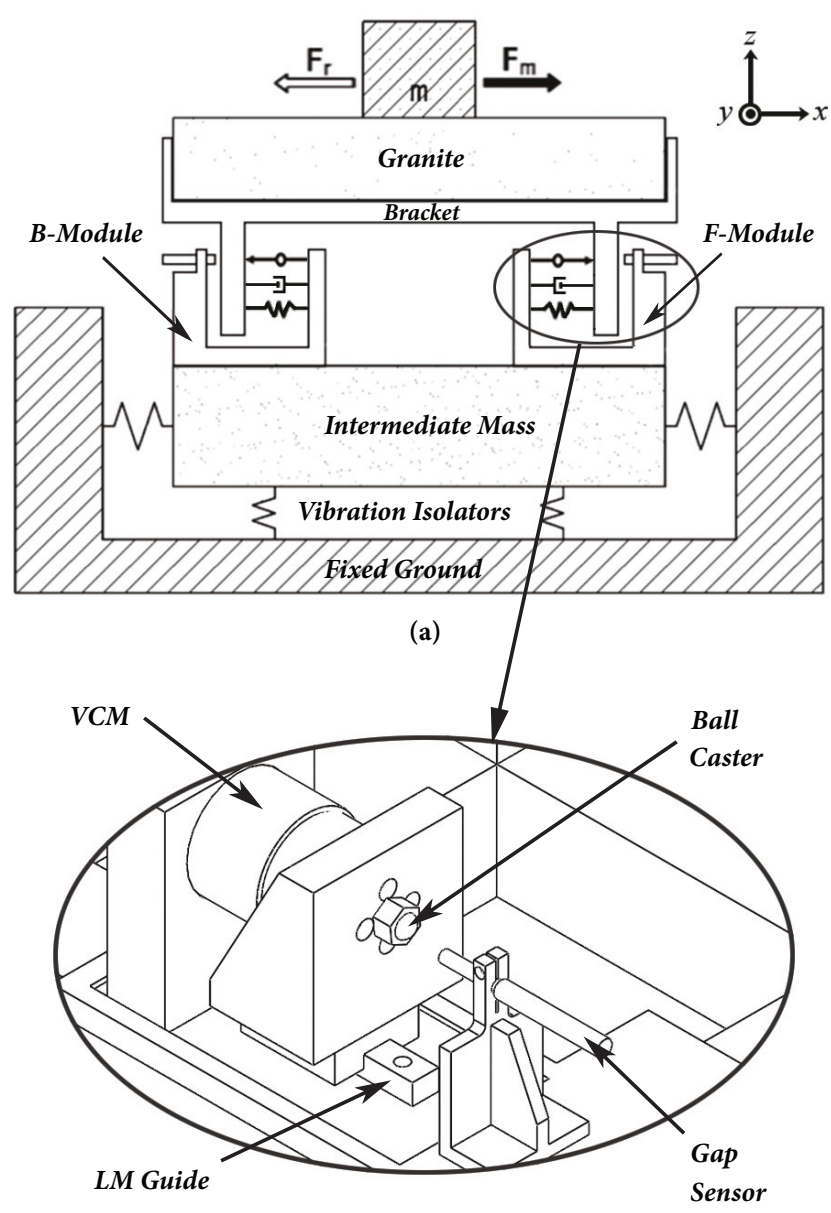

(b)

FIGURE 4: Reaction force compensator (a) in sectional schematic view and (b) its detail.

generate a peak force that exceeds the summation thrust force and to produce a continuous force that exceeds the sum of the preload and friction of the LM guide.

The thrust force of the linear motor is $325 \mathrm{~N}$ from its specifications. This is the compensation needed by the reaction force. The inertial force is given by (1). This is required for feedback control of the VCM.

$$
F_{r e q}=(2 \pi f)^{2} m d
$$

As shown in Figure 3, the resonant frequency $f$ is $2.5 \mathrm{~Hz}$ when using the air spring isolator. The mass of granite was 1,661 $\mathrm{kg}$, and the maximum displacement $d$ was $86 \mu \mathrm{m}$ from the time domain response in Figure 2(b). Therefore, the inertial force calculated from the equation was $36 \mathrm{~N}$. The above results indicated that the actuator required a peak force of $361 \mathrm{~N}$ to simultaneously perform the reaction force compensation and feedback control. An offset VCM force to keep the contact is required. The force plays a role of preload. The friction was very small when compared with the peak force; therefore, it was excluded from the estimation.

Specifically, AVM90-HF-10 from Akribis was used as the VCM actuator, and its specifications are listed in Table 3. 


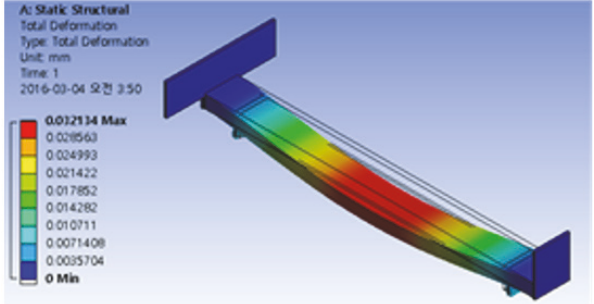

(a)

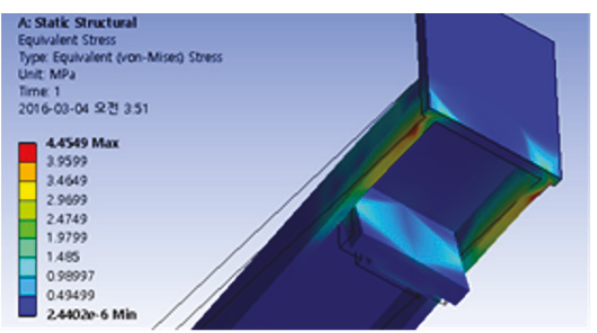

(b)

FIGURE 5: Result of finite elements analysis for bracket with respect to (a) deformation and (b) equivalent stress in SUS304.

TABLE 3: Specifications of VCM for RFC.

\begin{tabular}{lcc}
\hline Item & Unit & Specification \\
\hline Stroke & $\mathrm{mm}$ & 10.0 \\
Continuous force $\left(@ 100^{\circ} \mathrm{C}\right)$ & $\mathrm{N}$ & 153 \\
Peak force & $\mathrm{N}$ & 611 \\
Gap between coil \& magnet & $\mathrm{mm}$ & 0.650 \\
Mechanical time constant & $\mathrm{ms}$ & 1.46 \\
\hline
\end{tabular}

TABLE 4: Specifications of noncontact displacement sensor (gap sensor).

\begin{tabular}{lcc}
\hline Item & Unit & Specification \\
\hline Sensing range & $\mu \mathrm{m}$ & 1,000 \\
Bandwidth & $\mathrm{Hz}$ & 1,000 \\
RMS distance noise & $\mathrm{nm}$ & 2.90 \\
Resolution & $\mathrm{nm}$ & 5.70 \\
Output signal & $\mathrm{V}$ & \pm 10.0 (Analog) \\
\hline
\end{tabular}

Peak force specification of the actuator corresponded to 611 $\mathrm{N}$ and the thrust force was $69 \%$ larger when compared to the required force. The 5503 model from Microsense was used as a noncontact displacement sensor (gap sensor) for control, and its specifications are listed in Table 4.

There could be problems in system precision and safety if excessive deformation occurs in the bracket due to static load or resonance, because the actuating force of the VCMs is transferred to the granite through the bracket. The other mechanisms in Figure 4 are short and thick compared to the bracket. Therefore, they are considered relatively rigid. Thus, a design plan was verified through finite element (FE) analysis using the ANSYS 16.2 when the bracket material and size were selected.

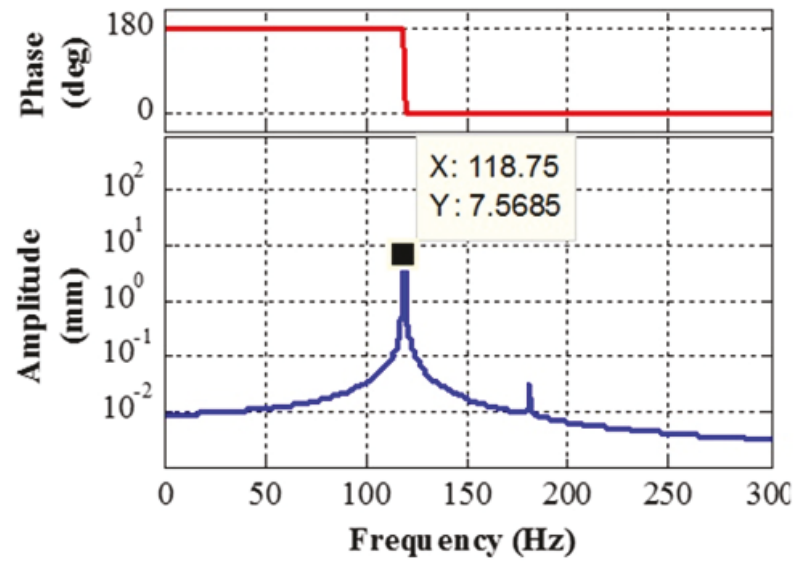

FIgURE 6: Harmonic response using finite elements analysis for AL6061.

FE models were made based on the candidate material, SUS304, for the maximum load assumption. The maximum load was from the bracket's own weight and the maximum output force of the VCMs. The maximum output does not apply in actual operation; however, the worst case of failure is considered in the model. A mesh with 6,622 nodes and 1,636 elements and with a size of about $530 \mathrm{~mm} \times 252 \mathrm{~mm} \times 1243$ $\mathrm{mm}$ was generated in the FE model. The displacement on the face fixed to the granite with the bolt was constrained to zero.

Figure 5 depicts the static analysis to verify safety under the maximum load assumption. Figure 5(a) shows the analysis of the SUS304 material. The maximum displacements corresponded to $32 \mu \mathrm{m}$ for the SUS304 materials. The maximum internal stress was $4.45 \mathrm{MPa}$ and this corresponded to $2.07 \%$ of the yield strength of $215 \mathrm{MPa}$ [15]. Figure 5(b) shows the results of the stress analysis.

With respect to the dynamic load due to resonance or repeated actuation, harmonic response analysis was used to evaluate the deformation under the dynamic load. The natural frequencies in Figure 6 corresponded to $119 \mathrm{~Hz}$. The structure did not exhibit resonance near the frequency of interest below $5 \mathrm{~Hz}$. From the results, the displacements at an excitation of the natural frequency corresponded to 7.57 $\mathrm{mm}$. Despite the simplified FE model that did not consider a damping coefficient, it is reasonable to assume that the SUS304 material was suitable for ensuring stability under both the static and repetitive load.

\section{Control Algorithm for Reaction Force Compensator}

In order to cope with various situations, the reaction force due to the acceleration and deceleration of the linear motion on the granite was considered as a disturbance. Because the pneumatic valve control of the isolator is independent of the main controller, it is difficult to control the displacement of the granite after it starts to move. Likewise, the effort to cancel the disturbance based on a feedback control scheme is ineffective to suppress incipient movement caused by the reaction force. The proposed mechanism in Figure 4 can be 


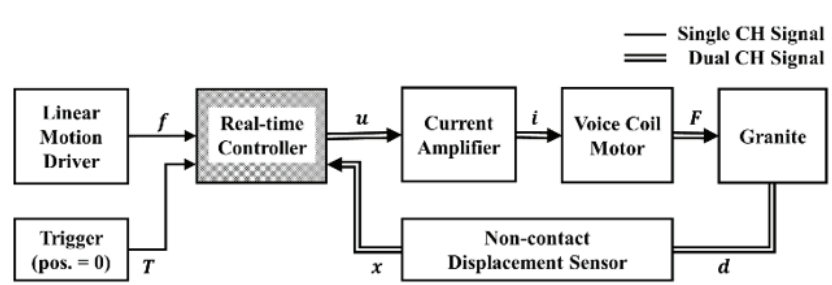

FIGURE 7: Control system for the reaction force compensator (RFC).

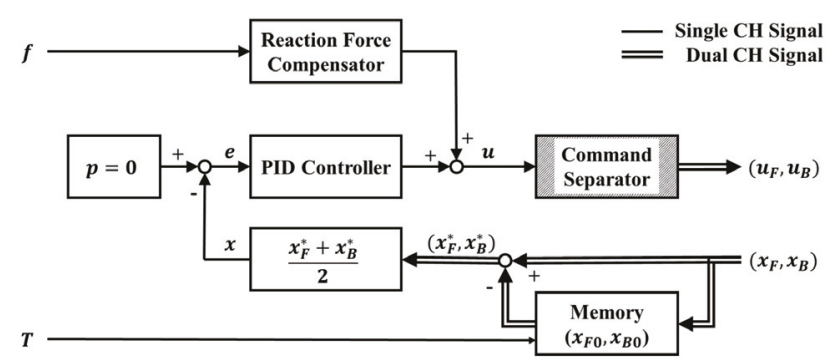

FIGURE 8: RFC control algorithm implemented with real-time controller.

operated immediately using the command signal of the linear motor.

The configuration of the control system for real-time control of the RFC is depicted in Figure 7. $f$ is the command signal of the linear motor from the linear motion driver. $u$ is the command output with analog voltage. $i, F, d$, and $x$ are current from the amplifier, generated force by VCM, displacement of the granite, and feedback signal with analog voltage, respectively. The present state of the granite's position is considered the initial position of control algorithm when the trigger signal $T$ is generated by the user's command.

The linear motion driver is a control unit for the linear motor as shown in Table 2. DS1005 from dSPACE with a 16-bit analog-to-digital convertor was used as the real-time controller. The voice coil motors in Table 3 are used for the actuator with a current amplifier. The gap sensors in Table 4 are used to measure the displacement of the granite. Both the F- and B-modules have a connection to another component with an independent communication channel. In Figures 7 and 8 , the dual channel means that the F- and B-modules have their own loop for signal routing.

Figure 8 shows a block diagram of the control algorithm for RFC which is the subsystem of the real-time controller in Figure 7. $p$ is the desired position of the granite that is fixed to zero. $e$ is the position error of the granite as an input command for the PID controller. $u_{F}, u_{B}, x_{F}$, and $x_{B}$ are the command voltages for the F- and B-modules and the feedback voltage from the displacement sensor. The current position is a relative value with respect to the memorized initial position when the trigger is turned on. The arithmetic mean values of $x_{F}$ and $x_{B}$ are used to calculate $e$ for the feedback control algorithm.

The control algorithm can be divided into the reaction force compensation, feedback, and output parts. The realtime controller receives a command signal from the linear motion module and generates a command signal to compensate the reaction force with the VCM like a feedforward controller, because the reaction force of the linear motor is the major disturbance to the granite in the precision stage. The output signal of the reaction force compensation can be expressed as follows:

$$
u=\frac{K_{V}}{K_{M}} v+K_{P} e+K_{I} \int_{0}^{t} e d \tau+K_{D} \frac{d e}{d t} .
$$

Here $K_{V}, K_{M}$, and $v$ in the first term, which is for the reaction force compensation, denote the voltage-force ratio of the VCM amplifier, voltage-force ratio of the linear motion module, and force command signal of the linear motion module controller, respectively. The last three terms are for the PID control with the feedback signal from the signal averaging block.

Additionally, $K_{V}$ and $K_{M}$ were considered as constants due to the linearity in the operational range in which $K_{V}=$ $325 \mathrm{~N} / \mathrm{V}$ and $K_{M}=61.1 \mathrm{~N} / \mathrm{V}$ from the specifications of the linear motion driver and VCM amplifier. Hence, the RFC was implemented with a fixed gain, which was the amplification ratio $K_{V} / K_{M}=5.32$.

A homing algorithm was also introduced in the RFC as shown in Figure 8. The noncontact displacement sensor used in the reaction force compensator has its own home position because it measures absolute displacement. However, a displacement of a few tens of micrometers could occur due to the deflection of beam structure as well as a slight deviation in the VCM. Therefore, a process to home the current location to zero was necessary prior to the initialization of the stage operation command. The current location was saved in the dSPACE memory through an external trigger $T$ to configure an algorithm that redesignated the starting point. Generally, the trigger signal is set once before the operation of the entire LDI process.

For convenience, the two displacement sensors faced opposite directions in which the module with the sensor direction identical to the linear motion coordinates was referred to as the F-module. The opposite module was referred to as the B-module. A direction gain of -1 was added in the signal averaging step because the B-module outputs a negative thrust against that in the positive direction of linear motion.

In order to prevent the independent operation of F- and B-modules of the VCMs, a command separation was devised. The two modules used in the RFC were symmetric in opposite directions of the common direction of motion. Furthermore, each VCM actuator was designed to produce a unidirectional pushing force only in a specific direction. It was necessary to pay attention to the operation because an overload could be applied to the VCM actuator or the ball caster could lose the contact, if the two modules were independently actuated contrary to intention.

A subsystem that controlled the output was separately set to block these unintended scenarios. A block diagram of the command separator in Figure 8 is shown in Figure 9. $C_{P}$ in the block diagram denotes the constant for the ratio of preload to maximum actuating force. A value of 1 for the saturation 


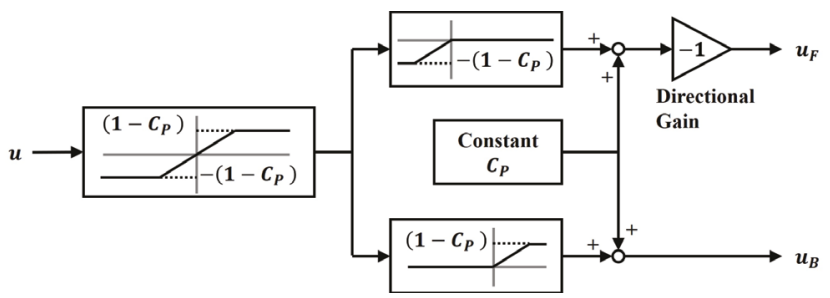

FIGURE 9: VCM command separator as a subsystem of the control algorithm.

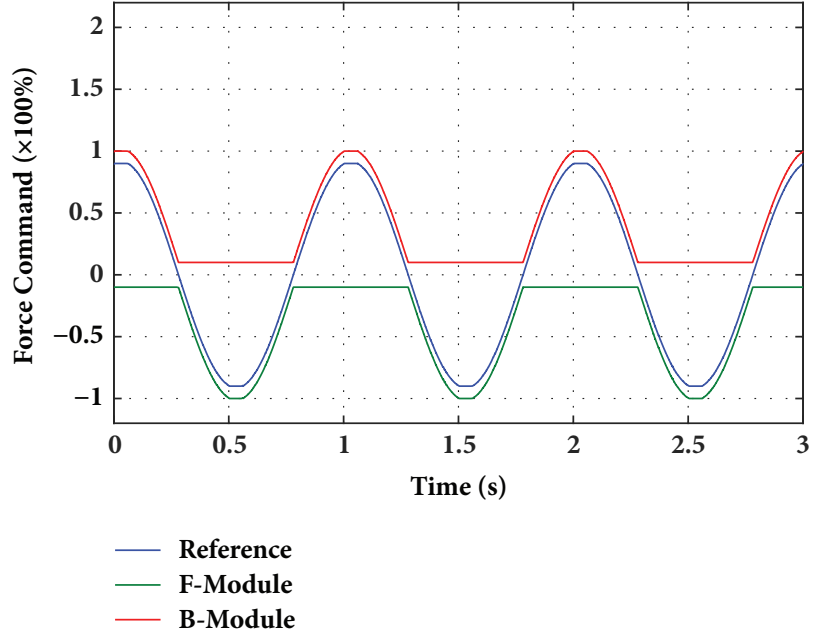

(a)

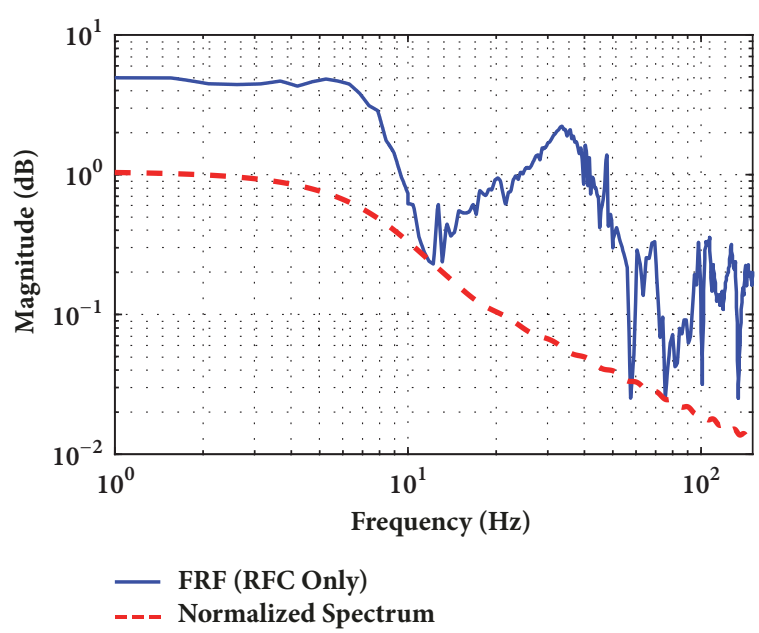

(b)

FIGURE 10: Verification of performance of actuation in (a) time domain and (b) frequency domain.

implies that the VCM output corresponded to $100 \%$. The F-module's sensor measures the module's motion, but the signal is opposite because of its setup in Figure 4(b). It was connected by directional gain (-1) in Figure 9 .

\section{Experiments}

In order to check the RFC operation, a sine input signal was applied in the output as shown in Figure 9 prior to the experiment for evaluation. The output signal was transferred to each actuator, and the displacement of granite was then measured. The preload related to $C_{P}$ in Figure 9 was decided by repetitive trial and error. The minimum $C_{P}$ was 0.1 without separation between ball caster and steel bracket for either Fmodule or B-module. It was observed in Figure 10(a) that a constant output signal at $10 \%$ of rated force was maintained for the preload and that the sum of the two signals followed the sine input signal.

The frequency response of the proposed mechanism with $K_{V} / K_{M}=5.32$ is illustrated with a blue line in Figure 10(b). The reference acceleration in Figure 2(a) is converted to a frequency range with a red dashed line in Figure 10(b). The frequency response of the reaction force compensator has much higher magnitude in comparison with the normalized spectrum of the main disturbance to cancel for most frequencies less than $100 \mathrm{~Hz}$. The critical point is found around $10 \mathrm{~Hz}$. However, the magnitude at $5.0 \mathrm{~Hz}$ is large enough to target that bandwidth. As shown in Figure 3(a), the reaction force of the linear motor does not easily affect the displacement of the granite at frequencies higher than $5.0 \mathrm{~Hz}$.

The performance of the proposed reaction force compensator is shown in Figure 11. The uncompensated case with a gray dashed line in Figure 11 is the same as in Figure 2(b). From the red line in Figure 11(a), it can be observed that the feedback control by the last three terms in (2) sufficiently reduced the difference. In particular, the relative position error of the stabilized state is closest to the initial position. However, the performance deteriorated in terms of maximum displacement when compared with that of the RFC, because the VCM's actuation was carried out after detection of errors. The delayed detection give the lagged response which results in the insufficient compensation.

In Figure 11(c), it can be observed that the size of the peaks was reduced to $39 \mu \mathrm{m}$ by only using the RFC. However, the generated force of the VCM that is synchronized with the driving of the linear motion module causes a displacement difference from zero, that is, a steady state error. These errors were due to the combination of the reaction force and the uncontrollable pitching motion of the granite. The rotated granite required a long time to stabilize because of the overdamped condition given by the low stiffness and high damping ratio of the air spring. The undesirable rotational motion had nonlinear behavior and an unpredictable magnitude, similar to backlash. 


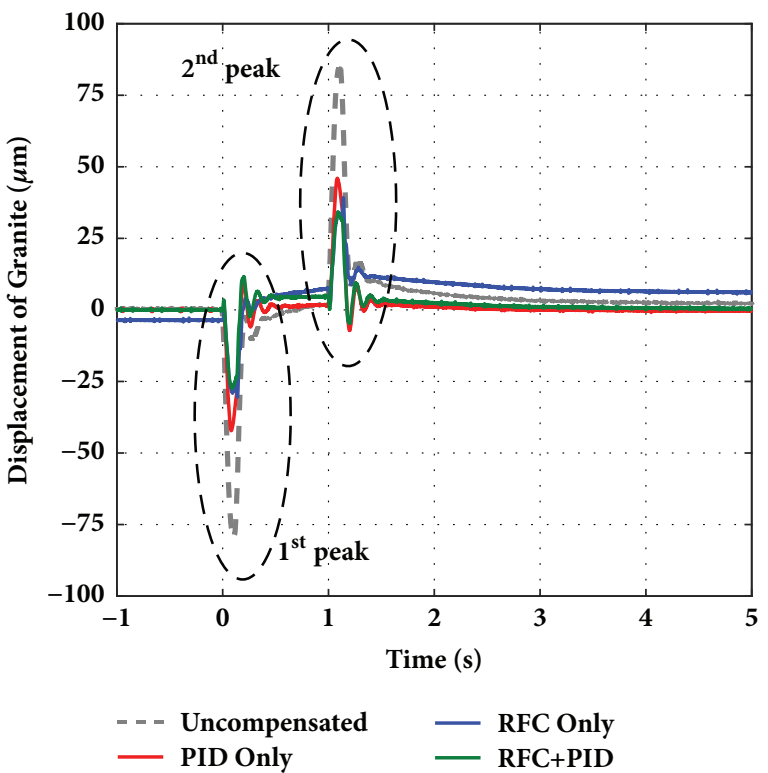

(a)

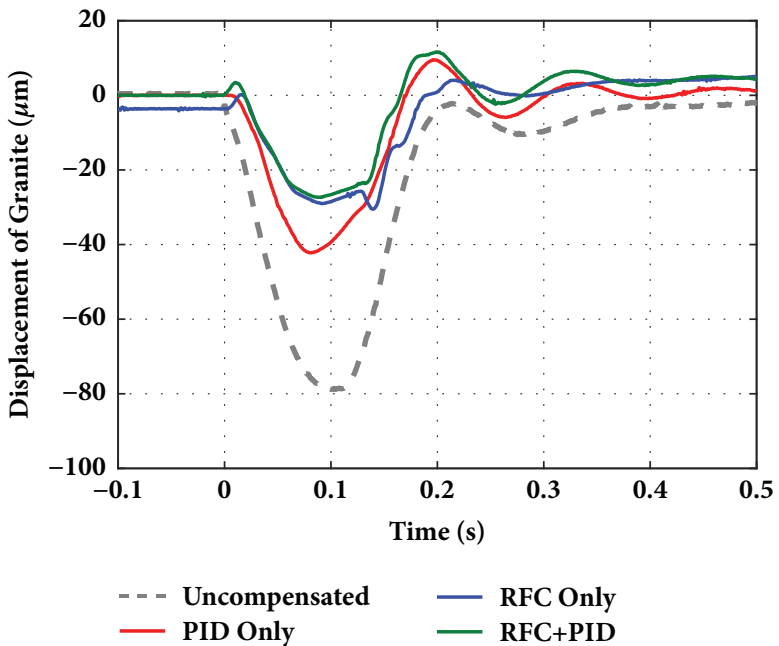

(b)

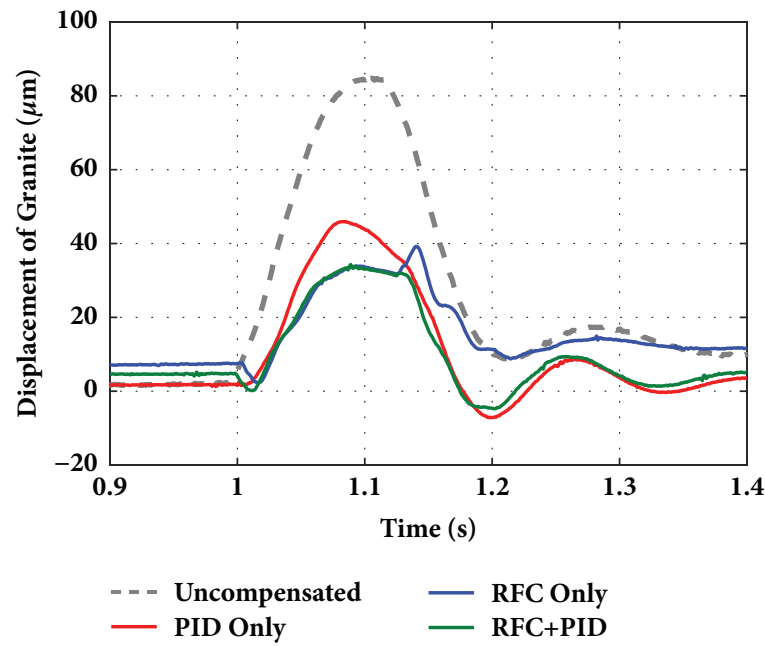

(c)

FiguRE 11: Experimental results of (a) the time response of the granite, (b) the magnified graph for $1^{\text {st }}$ peak, and (c) the magnified graph for $2^{\text {nd }}$ peak.

In spite of the steady state error, the RFC control scheme was more effective for the step-and-scan procedure of the LDI process. Because the resonance of the UV laser head is caused by the sudden movement of the granite, an angular error between the UV laser head and the workpiece may be calibrated in a steady state. In addition, no sensors are needed to apply the proposed RFC control scheme, in contrast to the previous studies [12-14].

The simultaneous use of the RFC and PID control resulted in a peak displacement difference of $34 \mu \mathrm{m}$, which corresponded to a reduction of approximately $60 \%$ when compared with the displacement difference of $86 \mu \mathrm{m}$ without the compensation. The steady state error and parasitic motion with RFC control can be suppressed with the feedback control. The combination of RFC and PID is recommended when the RFC control scheme shows insufficient performance caused by unpredictable disturbances.

\section{Conclusions}

In this study, a reaction force compensator to reject undesirable vibration of the precision stage for LDI was designed and verified. The results indicated that the maximum perturbation of the granite decreased by approximately $57 \%$ with the proposed mechanism and a simple control scheme. With feedback control, roughly $60 \%$ of the vibration level of the granite can be suppressed.

This proves that this concept of the reaction force compensator is effective in reducing the vibration and error, which can improve precision against adverse conditions of 
impulsive disturbance. With the results of this research, the complicated structure of the UV laser head can be simplified for miniaturization and lighter weight. To improve the performance of the RFC control scheme without sensors, nonlinear control schemes and model-based predictions will be considered in the future study.

\section{Conflicts of Interest}

The authors declare that there are no conflicts of interest regarding the publication of this paper.

\section{Acknowledgments}

This work was supported by the National Research Foundation of Korea (NRF) grant funded by the Korea Government (MSIT) (no. NRF-2018R1A2B2002683).

\section{References}

[1] K. Jain, M. Klosner, M. Zemel, and S. Raghunandan, "Flexible electronics and displays: high-resolution, roll-to-roll, projection lithography and photoablation processing technologies for high-throughput production," Proceedings of the IEEE, vol. 93, no. 8, pp. 1500-1510, 2005.

[2] C.-W. Lee and S.-W. Kim, "An ultraprecision stage for alignment of wafers in advanced microlithography," Precision Engineering, vol. 21, no. 2-3, pp. 113-122, 1997.

[3] K. Kim, Y.-M. Choi, B.-U. Nam, and M. G. Lee, "Dual servo stage without mechanical coupling for process of manufacture and inspection of flat panel displays via modular design approach," International Journal of Precision Engineering and Manufacturing, vol. 13, no. 3, pp. 407-412, 2012.

[4] M. H. Kim, J. H. Jeong, H. Y. Kim, and D. G. Gweon, "A sixdegree-of-freedom magnetic levitation fine stage for a high-precision and high-acceleration dual-servo stage," Smart Materials and Structures, vol. 24, Article ID 105022, 2015.

[5] S. J. Lee, J. S. Kim, B. C. Shin, D. W. Kim, and M. W. Cho, "Development of an LDI system for the maskless exposure process and energy intensity analysis of single laser beam," Journal of the Korean Society of Manufacturing Technology Engineers, vol. 19, pp. 834-840, 2010.

[6] O. Yavas and M. Takai, "Effect of substrate absorption on the efficiency of laser patterning of indium tin oxide thin films," Journal of Applied Physics, vol. 85, no. 8 I, pp. 4207-4212, 1999.

[7] C. Choi, L. Lin, Y. Liu et al., "Flexible optical waveguide film fabrications and optoelectronic devices integration for fully embedded board-level optical interconnects," Journal of Lightwave Technology, vol. 22, no. 9, pp. 2168-2176, 2004.

[8] D. C. Nguyen and H.-J. Ahn, "A Fuzzy-P controller of an active reaction force compensation (RFC) mechanism for a linear motor motion stage," International Journal of Precision Engineering and Manufacturing, vol. 16, no. 6, pp. 1067-1074, 2015.

[9] M. M. Z. Shahadat, T. Mizuno, Y. Ishino, and M. Takasaki, "Active vibration isolation using negative stiffness and displacement cancellation controls: Comparison based on vibration isolation performance," Control Engineering Practice, vol. 37, pp. 55-66, 2015.

[10] C. H. Seo, H. G. Lee, Y. H. Jeon, and M. G. Lee, "A study on vibration reduction mechanism of precision stage for laser direct imaging," Journal of the Korean Society for Precision Engineering, vol. 34, no. 6, pp. 431-437, 2017.
[11] N. C. Singer and W. P. Seering, "Preshaping command inputs to reduce system vibration," Journal of Dynamic Systems, Measurement, and Control, vol. 112, no. 1, pp. 76-82, 1990.

[12] G. Pin, "A direct approach for the frequency-adaptive feedforward cancellation of harmonic disturbances," IEEE Transactions on Signal Processing, vol. 58, no. 7, pp. 3523-3530, 2010.

[13] K. Matsuda and S. Kijimoto, "Reaction disturbance cancellation for a magnetically levitated ladder arm of clean-room robot," in Proceedings of the 36th Annual Conference of the IEEE Industrial Electronics Society, IECON 2010, pp. 1596-1602, Glendale, AZ, USA, November 2010.

[14] H. T. Kim, A. M. Jeong, H. Y. Kim et al., "Lateral vibration control of a precise machine using magneto-rheological mounts featuring multiple directional damping effect," Smart Materials and Structures, vol. 27, no. 3, Article ID 037001, 2018.

[15] H. E. Boyer and T. L. Gall, Metals Handbook, American Society for Metals, Ohio, USA, 1985. 


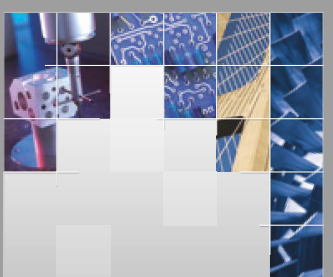

\section{Enfincering}
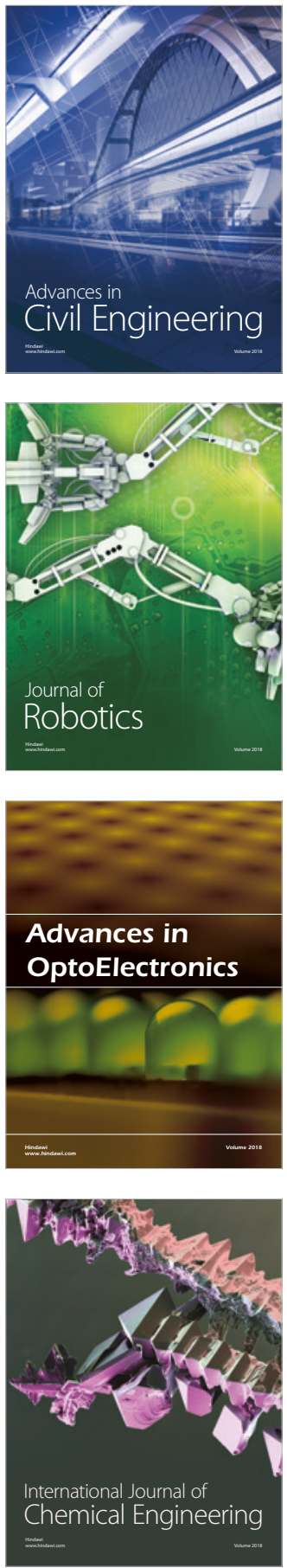

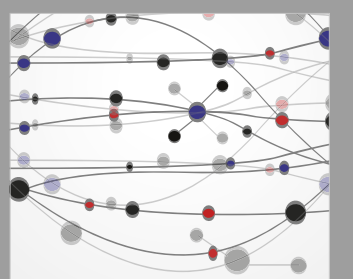

\section{Rotating \\ Machinery}

The Scientific World Journal

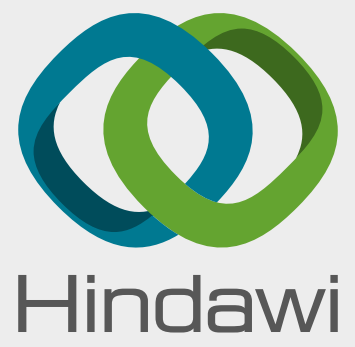

Submit your manuscripts at

www.hindawi.com
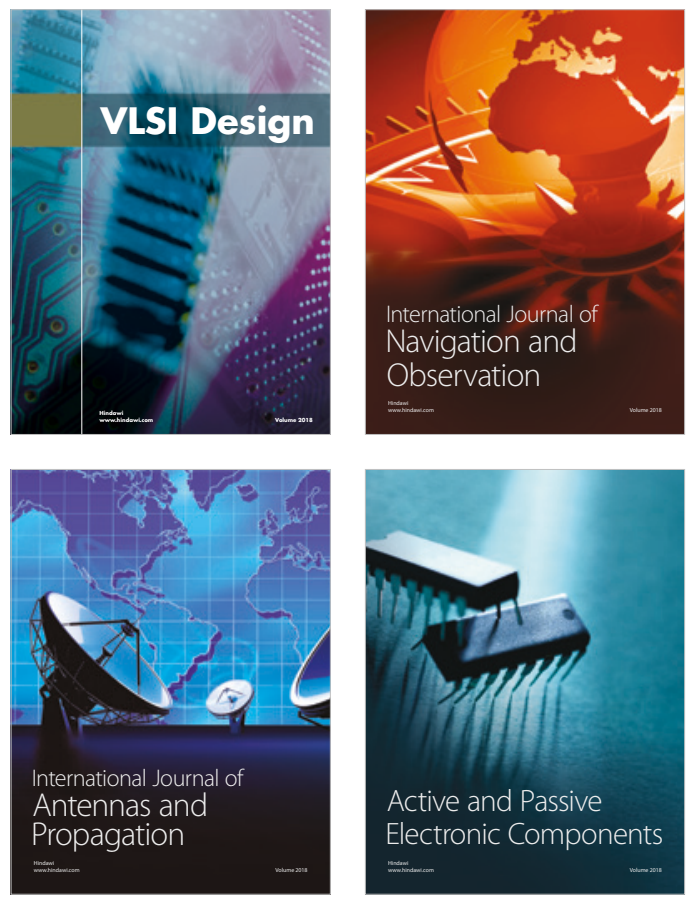
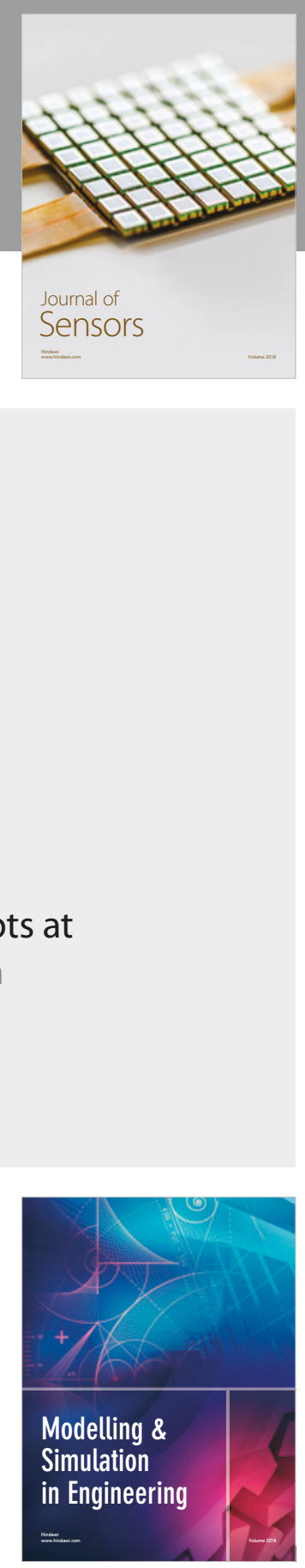

\section{Advances \\ Multimedia}
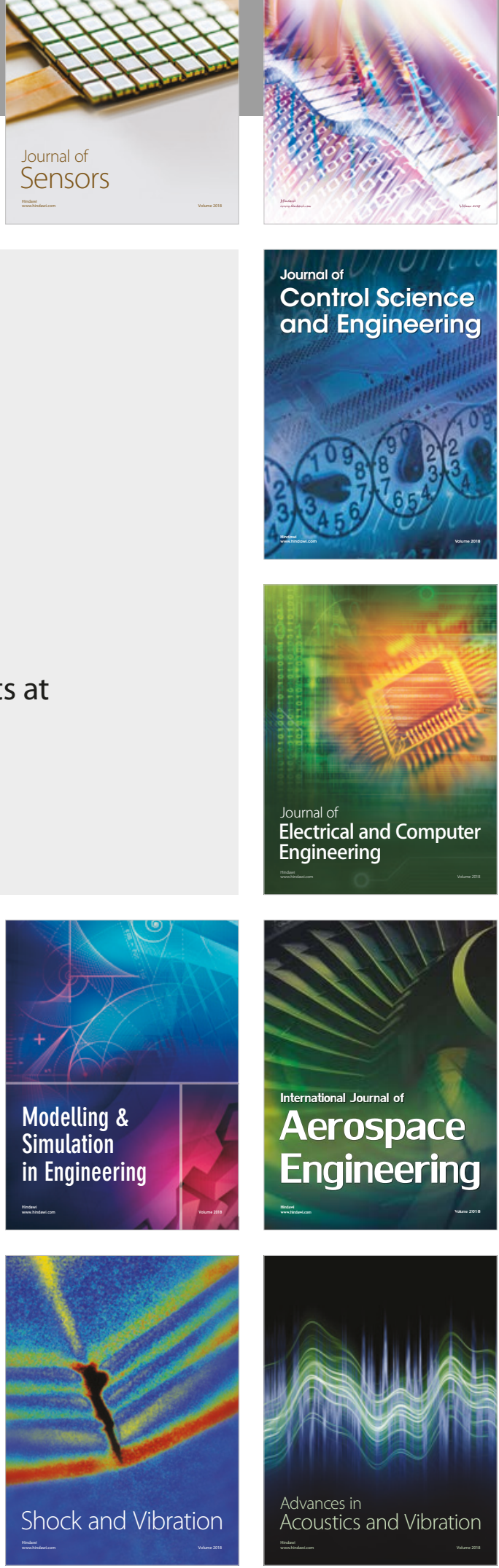